\title{
In-Situ Low Energy Ion Milling with a FIB-SEM for TEM lift-out Sample Preparation of Copper Damascene Structures Fabricated with Low k porous SiLK ${ }^{\mathrm{m}}$ Semiconductor Dielectric
}

\author{
E. R. Beach*, S. J. Rozeveld*, C. J. Wood*, M. V. Moore**, J. Waeterloos*** \\ * The Dow Chemical Company, Analytical Sciences, 1897 Building, Midland, MI 48667 \\ ** FEI Company, 7451 NW Evergreen Parkway, Hillsboro, OR 97124 \\ *** The Dow Chemical Company, on assignment at the Interuniversity Microelectronics Center (IMEC), \\ Leuven, Belgium
}

Porous ultra low $\mathrm{k}$ dielectric materials with copper $(\mathrm{Cu})$ interconnects and barrier materials are currently being investigated to meet expected demands for the 100 and $65 \mathrm{~nm}$ technology nodes defined in the International Technology Roadmap for Semiconductors (ITRS) [1].

ITRS requirements for porous ultra low $\mathrm{k}$ materials include a closed pore system, uniform pore distribution, and a narrow pore size distribution for each technology node [1]. Several techniques such as small angle x-ray scattering (SAXS), positron annihilation lifetime spectroscopy (PALS), and transmission electron microscopy (TEM) are currently used to characterize the pore size, distribution and degree of interconnectivity. Ultra low $\mathrm{k}$ materials routinely have pore sizes of $<10 \mathrm{~nm}$ and TEM analysis is markedly improved if thinner samples can be fabricated (e.g. thickness less than five times the pore diameter). A focused ion-beam (FIB) is the primary tool for preparing TEM sample from devices containing low $\mathrm{k}$ dielectric materials and copper [2]. A FIB-based technique using low energy $(10 \mathrm{kV}) \mathrm{Ga}$ ion milling has been evaluated to routinely prepare ultrathin TEM specimens $(<50 \mathrm{~nm}$ thick) with less surface damage than conventional FIB techniques. This technology is critical for the development and integration of porous low $\mathrm{k}$ materials and requires only $5-10$ minutes of additional sample preparation time using the FEI Strata DB235 focused ion beam-scanning electron microscope (FIB-SEM) [3].

Figures 1 and 2 show a substantial improvement in the TEM image quality after $10 \mathrm{kV}$ Ga ion milling, as compared to $30 \mathrm{kV}$ Ga ion milling, in two generations of developmental porous SiLK resin (samples with average pore sizes of 22 and $10 \mathrm{~nm}$, respectively). After low $\mathrm{kV}$ milling, both the Ta barrier layer and pore size in the porous SiLK films were more distinct in the TEM images as the overall sample thickness decreased from $\sim 80$ to $45 \mathrm{~nm}$. A reduction in the Ga-L $\alpha$ signal was observed by energy dispersive spectroscopy (EDS) after low $\mathrm{kV}$ milling which indicates less surface damage (Figure 3). Both atomic force microscopy (AFM) and parallel electron energy loss spectroscopy (PEELS) were utilized to quantify the relative change in sample thickness after low $\mathrm{kV}$ milling. PEELS results showed that the relative difference in the porous SiLK resin and $\mathrm{Cu}$ thickness decreased slightly after low $\mathrm{kV}$ ion milling. However, no further decrease in the thickness of the Ta barrier layer was observed. TappingMode ${ }^{\mathrm{TM}}$ AFM images shown in Figure 4 illustrate the surface topography of samples after standard FIB and low $\mathrm{kV}$ ion milling and support the relative thickness changes in the sample determined by PEELS.

References

1. International Technology Roadmap for Semiconductors, 2001 Edition, Interconnect chapter, 5.

2. M. W. Phaneuf et al., Microsc. Microanal. 8 (Suppl. 2), 2002, 52-53.

3. M. V. Moore, Microsc. Microanal. 8 (Suppl. 2), 2002, 60-61.

${ }^{\text {TM }}$ SiLK is a registered trademark of The Dow Chemical Company. 

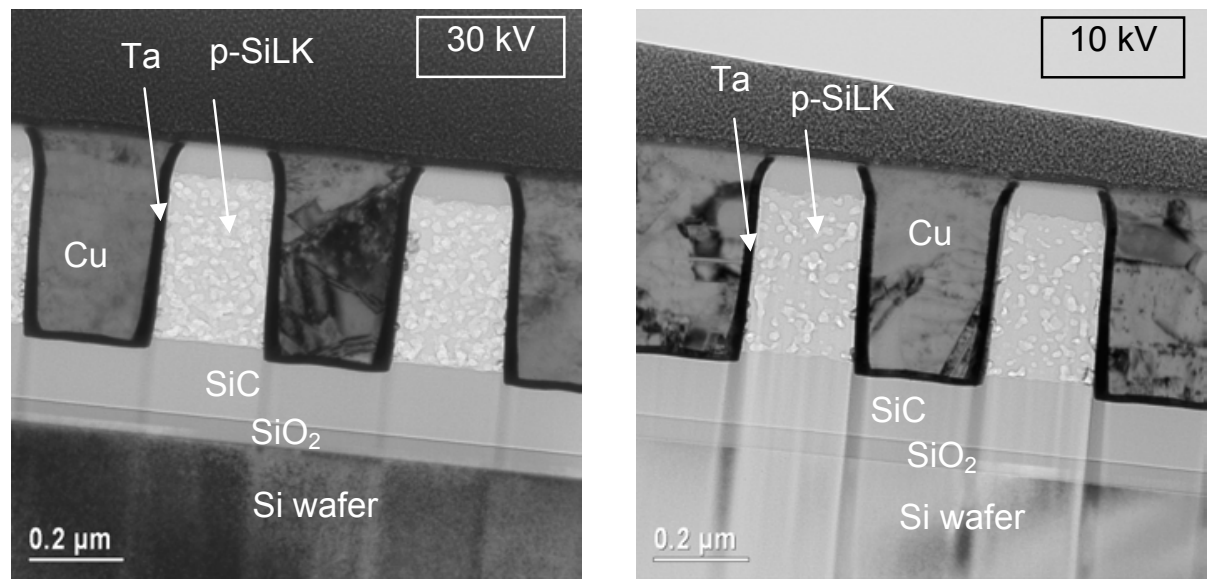

Figure 1. TEM images of lift-out samples from $\mathrm{Cu}$ damascene structure fabricated with developmental porous SiLK resin (version 7) with average pore size of 22nm. (Sample courtesy of IMEC)
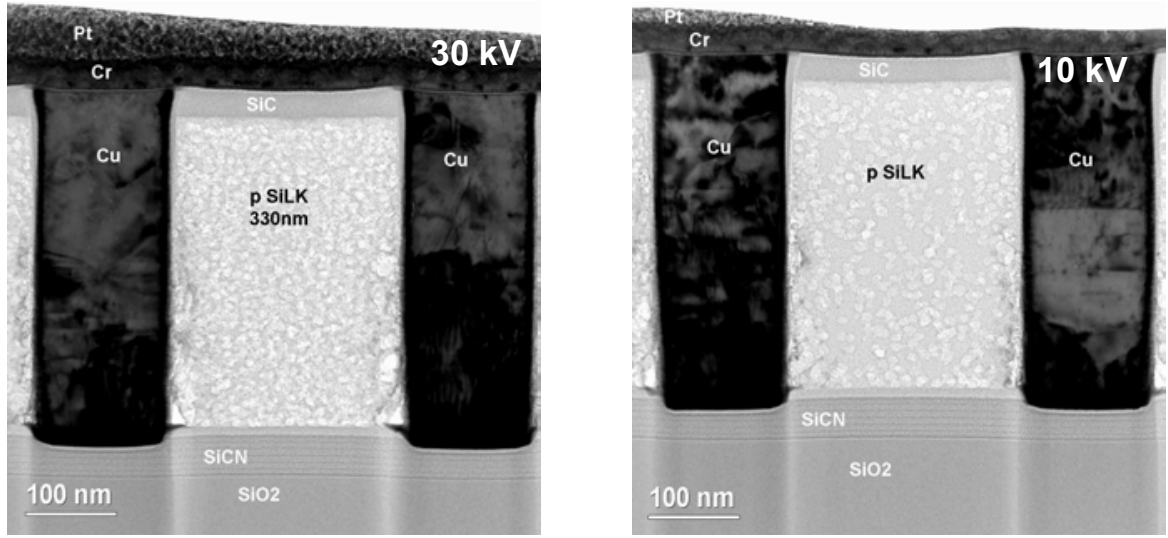

Figure 2. TEM images of lift-out samples from $\mathrm{Cu}$ damascene structure fabricated with developmental porous SiLK resin (version 9) with average pore size of $10 \mathrm{~nm}$.

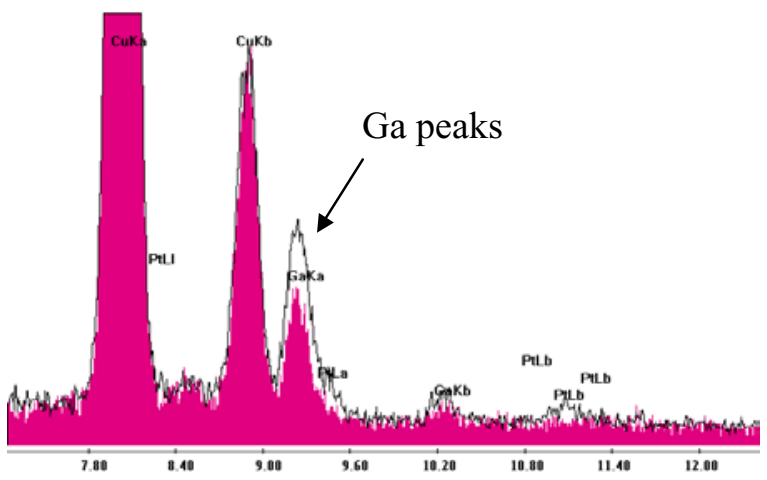

Figure 3. Comparison of EDS spectra from a $\mathrm{Cu}$ damascene lift-out sample cleaned with a $30 \mathrm{kV}$ $\mathrm{Ga}$ ion beam (black line) and a $10 \mathrm{kV} \mathrm{Ga}$ ion beam (red spectra). EDS spectra were recorded from $\mathrm{SiO}_{2}$. The $\mathrm{Cu} \mathrm{K} \alpha$ and $\mathrm{K} \beta$ peaks are due to stray electron scattering from the TEM grid.
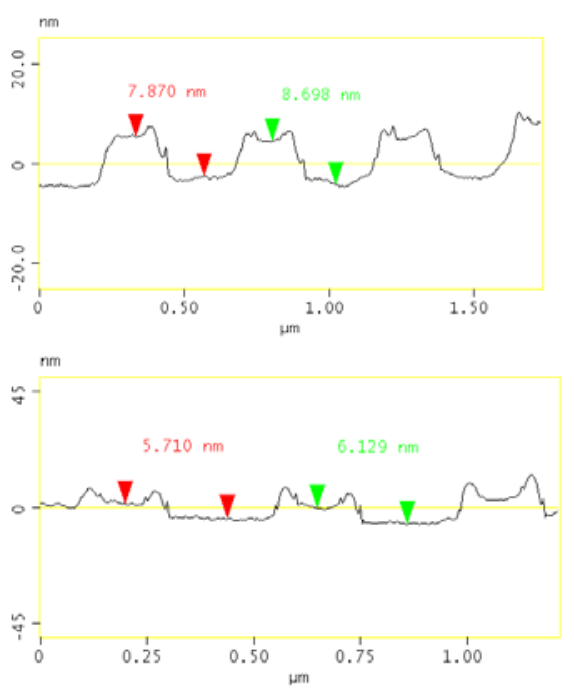

Figure 4. Cross-sectional AFM images of a $\mathrm{Cu}$ damascene lift-out sample after $30 \mathrm{kV}$ (top) and 10 $\mathrm{kV} \mathrm{Ga}$ ion milling. 